\title{
Neumoconiosis ocupacional por óxido de estaño
}

\section{Occupational pneumoconiosis due to tin oxide}

\author{
Luis Fernando Giraldo, Alirio Rodrigo Bastidas, Mateo Benavides, \\ Rafael García, Paulina Ojeda • Chía (Colombia)
}

\section{Resumen}

Se presenta el caso de hombre de 34 años con compromiso pulmonar secundario a exposición crónica a soldadura con óxido de estaño. El paciente consulto por tos seca de cuatro meses de evolución asociada a disnea leve y sibilancias ocasionales sin mejoría a tratamiento broncodilatador y con aparición de opacidades pulmonares en vidrio esmerilado hacia los lóbulos superiores, en biopsia transbronquial pulmonar se encuentran zonas de antracosis con macrófagos pardos sin fibrosis, hallazgos compatibles con estañosis. Esta enfermedad es poco frecuente, se relaciona con un curso benigno y debe ser reconocida dentro del grupo de enfermedades ocupacionales para definir el pronóstico del paciente. (Acta Med Colomb 2013; 38: xx-xx).

Palabras clave: óxido de estaño, alveolitis, neumoconiosis, enfermedad pulmonar intersticial, enfermedad ocupacional.

\section{Abstract}

A case of 34 year old man with lung involvement secondary to chronic exposure to welding with tin oxide. The patient consulted for a four-month history of dry cough associated with mild dyspnea and occasional wheezing without improvement with bronchodilator therapy and appearance of ground glass pulmonary opacities towards the upper lobes. In a transbronchial lung biopsy there are anthracosis areas with brown macrophages without fibrosis, findings consistent with stannosis. This is a rare disease that is associated with a benign course and should be recognized within the group of occupational diseases to define the prognosis of the patient. (Acta Med Colomb 2013; 38: xx-xx).

Keywords: tin oxide, alveolitis, pneumoconiosis, interstitial lung disease, occupational disease.

Dres. Luis Fernando Giraldo, Alirio Rodrigo Bastidas. Especialistas en Medicina Interna y Neumología: Servicio de Neumologia Clínica Universidad de La Sabana. Dr. Mateo Benavides, Dr. Rafael García: Internos Universidad de La Sabana. Dra. Paulina Ojeda. Especialista en Patología: Servicio de Patología, Hospital Santa Clara. Correspondencia: Dr. LuIs Fernando Giraldo. Clínica Universidad de La Sabana. Chía, Colombia.

Correspondencia.aliriorodrigo@yahoo.com Recibido: 20/XI/2012 Aceptado: 25/IX/2013

\section{Introducción}

La neumoconiosis por óxido de estaño es una entidad rara que hace parte del grupo de enfermedades pulmonares parenquimatosas difusas de origen ocupacional cuya característica fisiopatológica es producir un infiltrado celular inflamatorio con predominio de macrófagos cargados de nanopartículas de óxido de estaño sin que se produzca fibrosis pulmonar. Se decide presentar el caso de un paciente con hallazgos, clínicos, radiológicos e histopatológicos por este tipo de neumoconiosis, la cual debe ser diferenciada de otras enfermedades parenquimatosas e infecciosas de pronóstico diferente.

\section{Caso clínico}

Paciente de 34 años quien consultó por tos seca de cuatro meses de evolución, acompañada con expectoración hialina esporádica, disnea leve, sibilancias ocasionales y sudoración nocturna, como antecedente ocupacional se desempeña como soldador con óxido de estaño durante aproximadamente ocho años, los antecedentes de asma, tabaquismo y tuberculosis eran negativos. El paciente recibió manejo broncodilatador con beta- 2 agonistas, anticolinérgicos asî como con omeprazol por hallazgos endoscópicos de esofagitis grado A y pangastritis eritematosa superficial sin mejoría clínica.

Se había realizado previamente ecocardiograma transtorácico y TAC de senos paranasales con hallazgos normales.

Los signos vitales eran normales y no se encontraron hallazgos semiológicos patológicos al examen del sistema pulmonar y físico en general. Los laboratorios mostraron, cuadro hemático con hemoglobina de $16.1 \mathrm{~g} / \mathrm{dL}$, hematocrito de $47 \%$, leucocitos normales, gases arteriales sin 
desequilibrio ácido base, ni hipoxemia. La espirometría con FEV1/CVF 85\% VEF 100\%, CVF 97\%, baciloscopia seriada negativa. Radiografía tórax con opacidades de tipo reticulonodular de predominio parahiliar y central de ambos hemitórax (Figuras 1 y 2). Tomografía axial computarizada de tórax de alta resolución (TACAR) que evidencia áreas de vidrio esmerilado la mayoría de localización periférica y apical bilateral sin áreas de cavitación ni derrame pleural (Figuras 3 y 4).

Fibrobroncoscopia con árbol bronquial anatómicamente normal, no se detectaron lesiones, ni hemorragias, recuento diferencial con predominio de macrófagos en $78 \%$, linfocitos $10 \%$ y neutrófilos en $12 \%$, tinciones y cultivos para

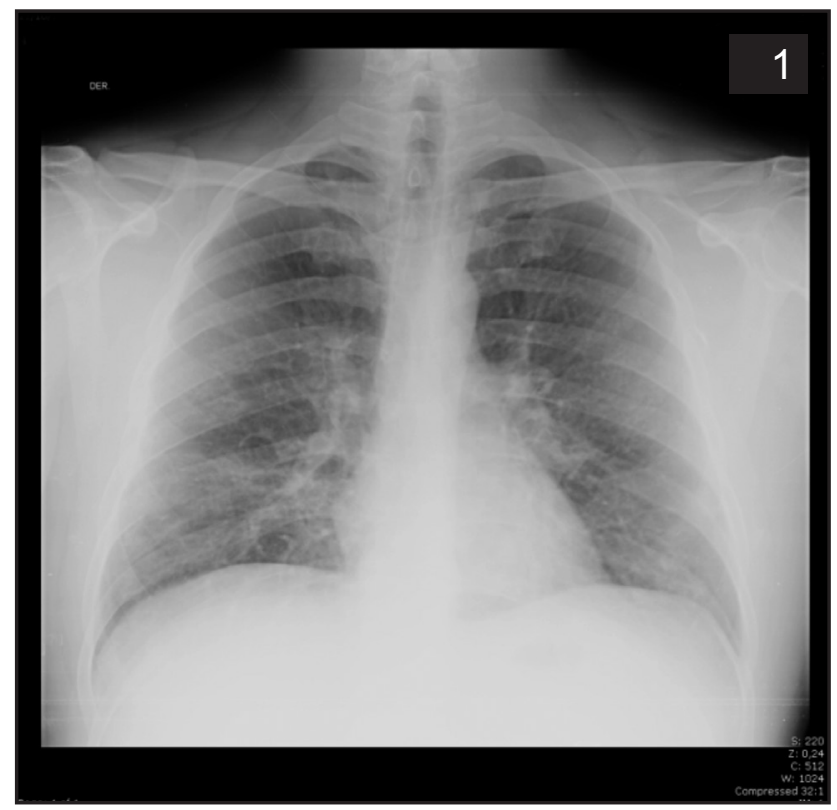

Figuras 1 y 2. Radiografía de tórax donde se observa infiltrado retinodular parahiliar.

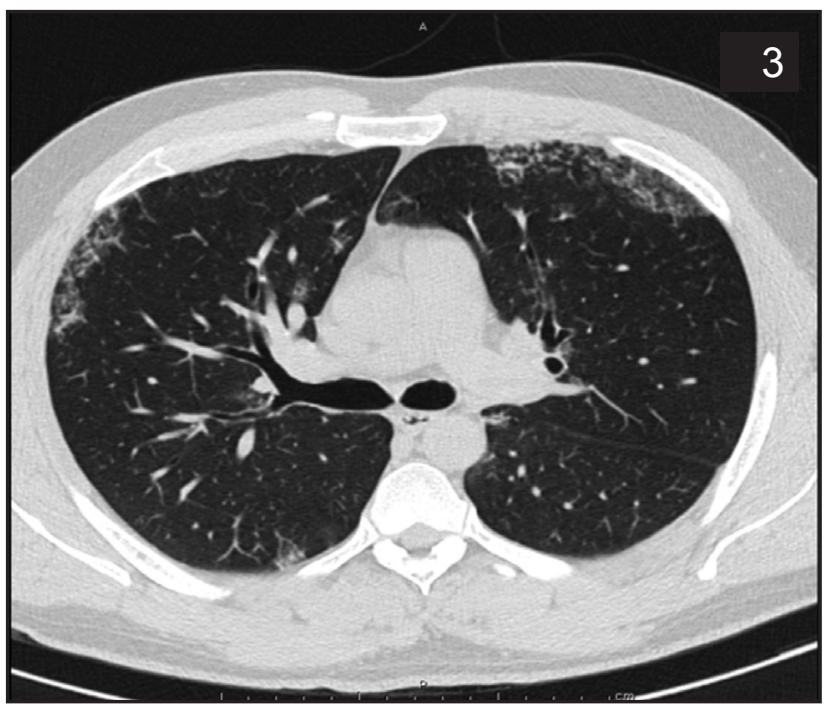

tuberculosis, hongos y gérmenes comunes negativos, la biopsia pulmonar transbronquial reportó gran cantidad de macrófagos pardos con pigmento antracótico sin evidencia de fibrosis o cristales (Figuras 5-7).

\section{Discusión}

La inhalación prolongada de partículas inorgánicas en lugares de trabajo se asocia a enfermedades pulmonares crónicas, cuya presentación clínica difiere según el agente exposicional $(1,13)$. La neumoconiosis por estaño es una enfermedad ocupacional producida por la inhalación de partículas inorgánicas de óxido de estaño. Es una enfermedad considerada de un curso benigno poco frecuente,
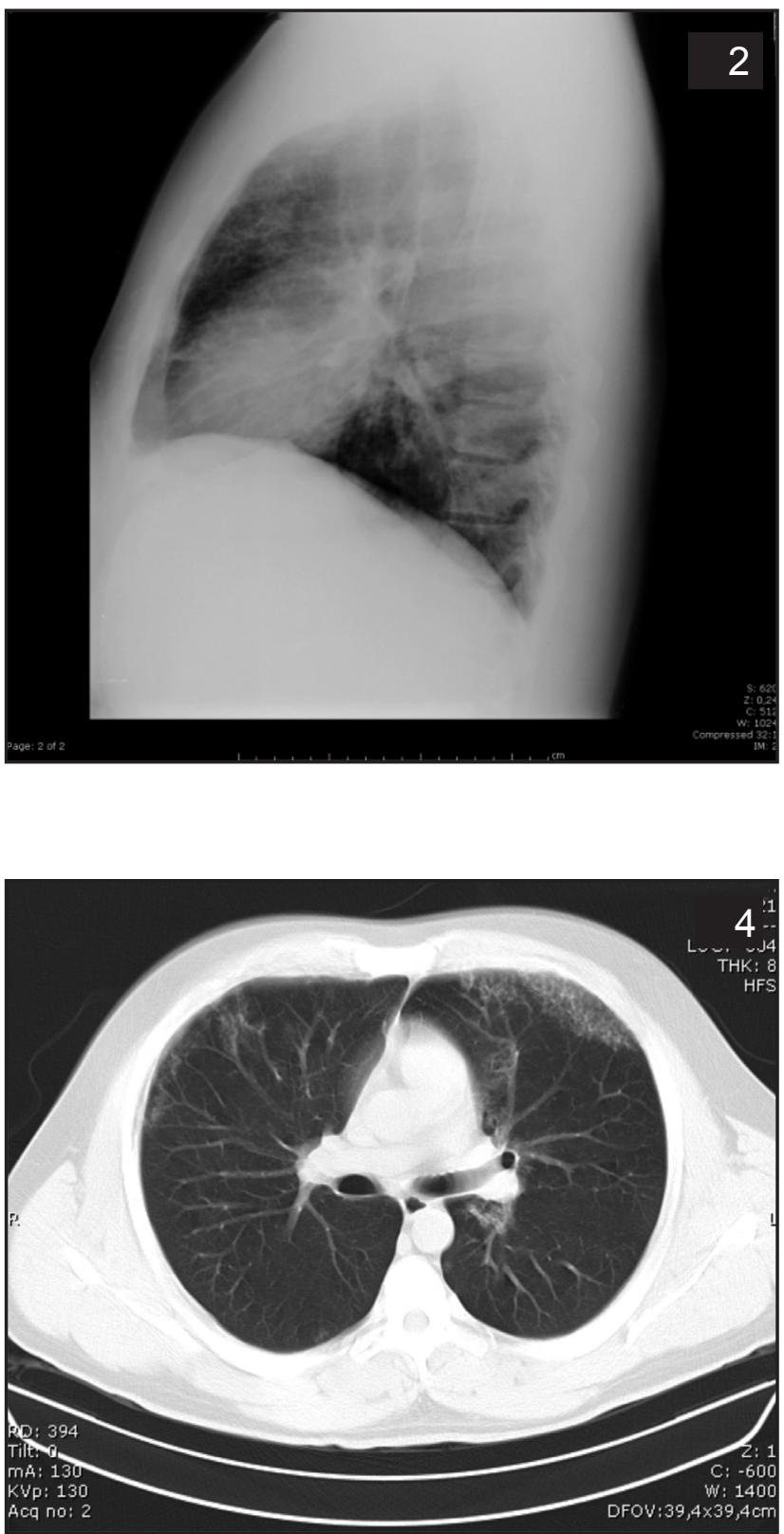

Figuras 3 y 4. TAC de tórax con áreas de infiltrado alveolar, con áreas de vidrio esmerilado de predominio en zonas superiores, en ambos hemitórax. 

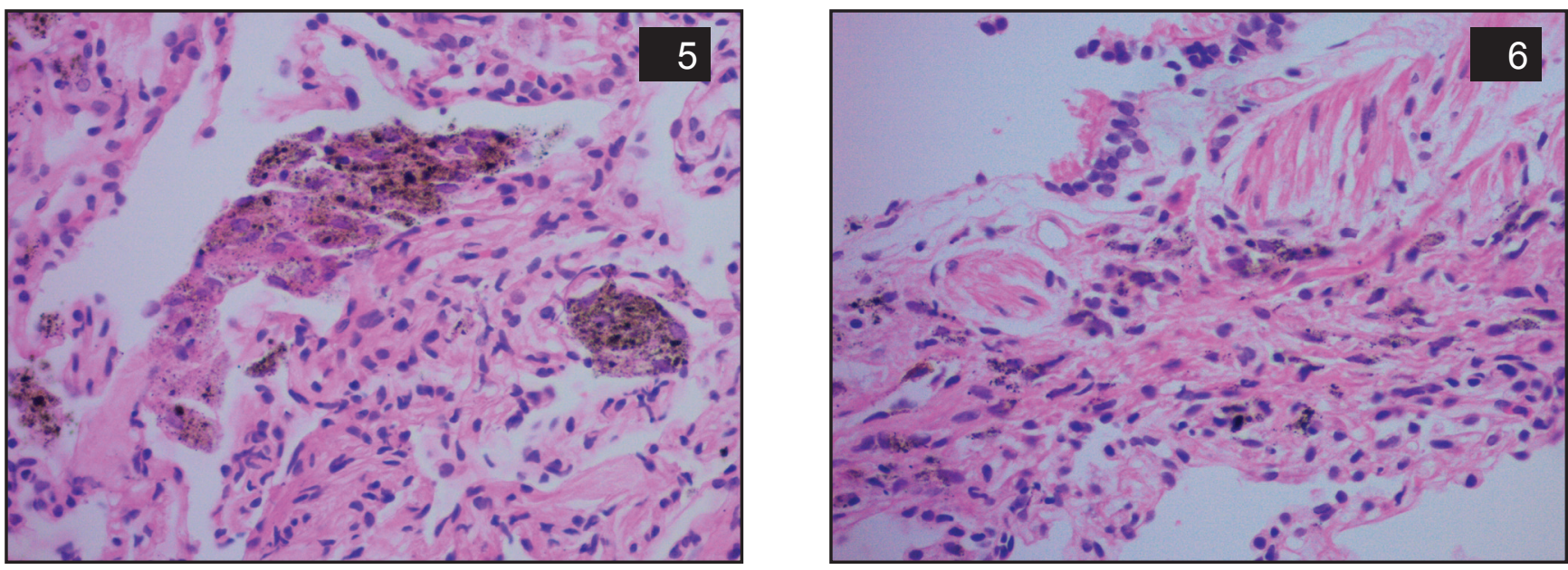

Figuras 5 y 6. Tejido de parénquima pulmonar y de la pared del bronquiolo donde se observan macrófagos con polvo de estaño en los alvéolos y en el intersticio. (H \& E a $40 x$ ).

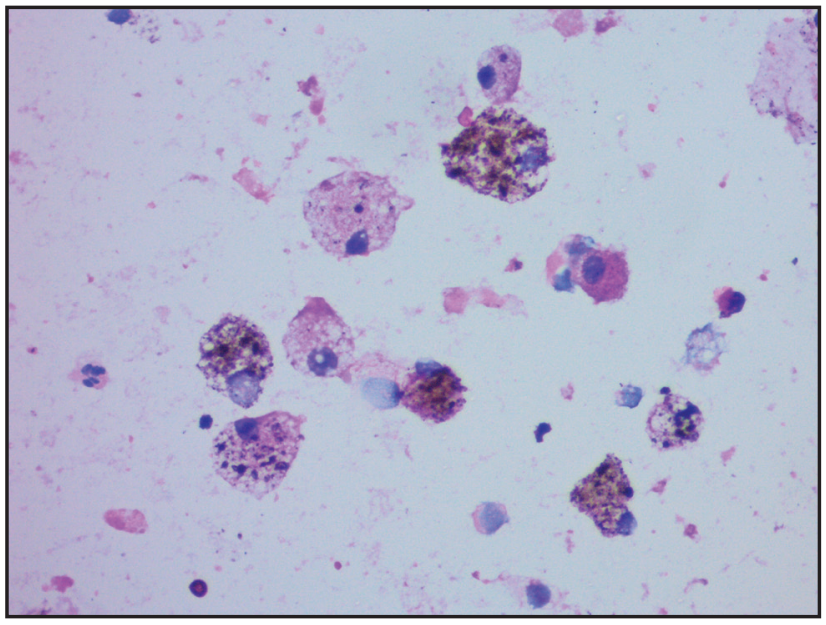

Figura 7. Lavado Bronquioloalveolar, se evidencia la presencia de macrófagos pardos cargados con pigmento antracotico $(H \&$ E $a 40 x)$.

que se presenta en pacientes que trabajan en operaciones de purificación del mineral, fundición, minería o soldadura en donde existe desprendimiento de nanopartículas de dicho polvo que al ser inhaladas por largos periodos de tiempo producen la enfermedad $(1,2)$.

En nuestro paciente la exposición crónica a la soldadura del óxido de estaño es el principal factor de riesgo para desarrollar la enfermedad, el tiempo de diez años se relaciona fuertemente con la aparición de esta entidad según lo reportado por Robertson y Schuler en varias series de casos donde el promedio de tiempo considerado para el desarrollo de la enfermedad fue de seis a ocho años de exposición (1, 3 , 4). La sintomatología de tos, disnea y sibilancias puede estar presente; sin embargo, los hallazgos pueden ser inespecíficos necesitando estudios complementarios para descartar otras posibilidades diagnósticas; el antecedente negativo para asma, consumo de cigarrillo y las tinciones especiales para microorganismos, TC de senos paranasales sin alteraciones hace poco probable la existencia de enfer- medades inflamatorias e infecciosas, la esofagitis puede ser causa de tos pero no se relaciona con el tipo de infiltrados pulmonares, además el tratamiento con omeprazol sin mejoría de los síntomas sugiere etiología diferente al reflujo como causa de tos.

La neumoconiosis por estaño se caracteriza por hallazgos radiográficos donde predominan los infiltrados difusos micronodulares, la mayoría en regiones superiores, como lo encontrado en la radiografía de tórax de nuestro caso. La tomografía de tórax de alta resolución demostró además aéreas de vidrio esmerilado (5-7). Estas alteraciones radiológicas no se relacionan con los resultados de las pruebas de función pulmonar, pudiéndose encontrar espirometrías y curvas flujo volumen normales $(1,3,4)$. El diagnóstico de nuestro caso se realiza mediante el estudio histopatológico donde se evidencia áreas de antracosis con macrófagos pardos cargados de pigmento antracótico y ausencia de fibrosis, sumado a los hallazgos radiológicos, y al antecedente ocupacional $(1,3)$.

Se ha documentado que los efectos deletéreos del estaño a nivel pulmonar y su pronóstico se basan fundamentalmente en reportes de casos con pobre evaluación de la exposición (2). El óxido de estaño, así como la exposición al sulfato de bario y la siderosis, se reconoce que producen un tipo de neumoconiosis no fibrótica, donde se encuentra gran cantidad de macrófagos, cargados con nanopartículas de estos compuestos (8). Los macrófagos al ser activados, liberan diferentes tipos de enzimas, citoquinas inflamatorias como el factor de necrosis tumoral (FNT) alfa, IL-1 y metabolitos del ácido araquidónico, que llevan a mayor reclutamiento de células inflamatoria al alvéolo, liberación de especies reactivas e inicio de estrés oxidativo lo que genera alveolitis, la cual puede reflejarse como áreas de micronódulos y vidrio esmerilado (9).

El grado de compromiso pulmonar dependerá de la cantidad, la solubilidad, la forma, el tamaño y la capacidad de depuración de las partículas retenidas en las vías aéreas (10). Así, probablemente el diámetro pequeño de 
las partículas de estaño, y su potencial de disolución se relacione con exposiciones de larga data sin generación de fibrosis (10). Se reconoce que las partículas de mayor tamaño con una mayor respuesta inflamatoria puede llegar a estimular fibroblastos intersticiales con producción de factores de crecimiento y aumento de factor de crecimiento transformante (TGF- $\beta$ ), importantes en la génesis de la fibrosis (10). Por otra parte, Borm sugiere que inhalación de grandes cantidades de estas nanopartículas puede desarrollar cuadro de injuria pulmonar aguda (11). Estudios experimentar en conejillos de indias, con inyecciones de óxido de estaño en peritoneo han experimentado cambios fibróticos; sin embargo, los reportes de caso en seres humanos donde se ha detectado fibrosis pulmonar también se han relacionado con infecciones concomitante de tuberculosis y exposiciones a asbesto y tabaco (12).

\section{Conclusión}

La soldadura por óxido de estaño puede comprometer el parénquima pulmonar y se debe tener en consideración dentro del diagnóstico diferencial de enfermedades ocupacionales de curso benigno.

\section{Referencias}

1. Recommendation from the Scientific Committee on Occupational Exposure Limits for tin and inorganic tin compounds, European Commission, Employment, Social Affairs and Inclusion SCOEL/SUM/97. November 2003 pag 5-9

2. Yilmaz A, Gocmen Ocal S, Doruk S, Acu B. Is tin fume exposure benign or not? Two case reports. Tuberk Toraks 2009; 57: 422-6.

3. Sluis-Cremer GK, Thomas RG, Goldstein B, Solomon A. Stannosis. A report of 2 cases. $S$ Afr Med J 1989; 75: 124-6.

4. Schuler P, Cruz E, Guijon C, et al. Stannosis: Benign pneumoconiosis owing to inhalation of tin dust and fume. Industrial Medicine Surgery 1958; 27: 432-5.

5. Byrne JD, Baugh JA. The significance of nanoparticles in Particle-induced pulmonary fibrosis. Mcgill J Med 2008; 11: 43-50.

6. Robertson AJ, Rivers D, Nagelschmidt G, Duncumb P. Stannosis: benign pneumoconiosis due to tin dioxide. Lancet 1961; 1: 1089-93.

7. Robertson AJ, Whitaker PH. Radiological changes in pneumoconiosis due to tin oxide. J Fac Radiol 1955; 6: 224-33.

8. Glazer CS, Newman LS. Occupational interstitial lung disease. Clin Chest Med 2004; 25: 467-478.

9. Güllï E, Karnak D, Kayacan O, Beder S. A tinner with stannosis and tuberculosis. Case Rep Clin Pract Rev 2005; 6: 73-76.

10. Li JJ, Muralikrishnan S, Ng CT, Yung LY, Bay BH. Nanoparticle-induced pulmonary toxicity. Exp Biol Med 2010, 235: 1025-1033.

11. Kumar V, Robbins S, Cotran R. Pathologic Basis of Disease, 8th ed. 2009 Saunders, Elsevier chapter 15.

12. Borm PJ. Particle toxicology: from coal mining to nanotechnology. Inhal Toxicol 2002; 14: 311.

13. Palacios DM, Méndez O. NeumopatÍa por humo de leña Un estudio en autopsias . Biomédica 1998; 153-60. 